\title{
Elevated D-dimers and lack of anticoagulation predict PE in severe COVID-19 patients
}

\author{
Basile Mouhat (10) ${ }^{1}$, Matthieu Besutti ${ }^{1}$, Kevin Bouiller ${ }^{2,3}$, Franck Grillet (10 ${ }^{4}$, \\ Charles Monnin ${ }^{1}$, Fiona Ecarnot ${ }^{1,5}$, Julien Behr ${ }^{4}$, Gilles Capellier ${ }^{5,6}$, \\ Thibaud Soumagne ${ }^{6}$, Sébastien Pili-Floury ${ }^{5,7}$, Guillaume Besch ${ }^{5,7}$, \\ Guillaume Mourey ${ }^{8,9}$, Quentin Lepiller ${ }^{10}$, Catherine Chirouze (10), \\ François Schiele $\mathbb{1}^{1,5}$, Romain Chopard ${ }^{1,5,11}$ and Nicolas Meneveau ${ }^{1,5,11}$
}

@ERSpublications

We studied predictors of pulmonary embolism in severe COVID-19 and found that D-dimer level and lack of any anticoagulant therapy were associated with a 17-fold and four-fold increase in PE, respectively, in COVID-19 patients with clinical signs of severity https://bit.ly/2ETfAfo

Cite this article as: Mouhat B, Besutti M, Bouiller K, et al. Elevated D-dimers and lack of anticoagulation predict PE in severe COVID-19 patients. Eur Respir J 2020; 56: 2001811 [https://doi.org/10.1183/ 13993003.01811-2020].

\section{ABSTRACT}

Background: Coronavirus disease 2019 (COVID-19) may predispose to venous thromboembolism. We determined factors independently associated with computed tomography pulmonary angiography (CTPA)confirmed pulmonary embolism (PE) in hospitalised severe COVID-19 patients.

Methods: Among all ( $\mathrm{n}=349)$ patients hospitalised for COVID-19 in a university hospital in a French region with a high rate of COVID-19, we analysed patients who underwent CTPA for clinical signs of severe disease (oxygen saturation measured by pulse oximetry $\leqslant 93 \%$ or breathing rate $\geqslant 30$ breaths $\cdot \mathrm{min}^{-1}$ ) or rapid clinical worsening. Multivariable analysis was performed using Firth penalised maximum likelihood estimates.

Results: 162 (46.4\%) patients underwent CTPA (mean \pm SD age $65.6 \pm 13.0$ years; $67.3 \%$ male (95\% CI 59.5$75.5 \%)$. PE was diagnosed in $44(27.2 \%)$ patients. Most PEs were segmental and the rate of PE-related right ventricular dysfunction was $15.9 \%$. By multivariable analysis, the only two significant predictors of CTPAconfirmed PE were D-dimer level and the lack of any anticoagulant therapy (OR 4.0 (95\% CI 2.4-6.7) per additional quartile and OR 4.5 (95\% CI 1.1-7.4), respectively). Receiver operating characteristic curve analysis identified a D-dimer cut-off value of $2590 \mathrm{ng} \cdot \mathrm{mL}^{-1}$ to best predict occurrence of PE (area under the curve $0.88, \mathrm{p}<0.001$, sensitivity $83.3 \%$, specificity $83.8 \%$ ). D-dimer level $>2590 \mathrm{ng} \cdot \mathrm{mL}^{-1}$ was associated with a 17 -fold increase in the adjusted risk of PE.

Conclusion: Elevated D-dimers $\left(>2590 \mathrm{ng} \cdot \mathrm{mL}^{-1}\right.$ ) and absence of anticoagulant therapy predict PE in hospitalised COVID-19 patients with clinical signs of severity. These data strengthen the evidence base in favour of systematic anticoagulation, and suggest wider use of D-dimer guided CTPA to screen for PE in acutely ill hospitalised patients with COVID-19.

This article has supplementary material available from erj.ersjournals.com

This article has an editorial commentary: https://doi.org/10.1183/13993003.03092-2020

Received: 15 May 2020 | Accepted after revision: 1 July 2020

Copyright OERS 2020. This version is distributed under the terms of the Creative Commons Attribution NonCommercial Licence 4.0. 


\section{Introduction}

Coronavirus disease 2019 (COVID-19), caused by severe acute respiratory syndrome coronavirus 2 (SARS-CoV-2), may predispose patients to venous thromboembolic (VTE) complications [1]. Preliminary reports suggest that the severe inflammatory response and other features of critical illness contribute to a procoagulant profile that predisposes to thrombotic events $[2,3]$. Possible forms of coagulopathy include haemostatic abnormalities, notably with increased levels of D-dimers [2-5].

Incident pulmonary embolism (PE) has been reported in 20.6-30.0\% of severe COVID-19 patients [6-10]. However, given the clinical presentation of patients with concomitant COVID-19 pneumonia, it is difficult to identify patients in whom PE should be suspected. In this regard, the indications for computed tomography pulmonary angiography (CTPA) remain to be defined [11-14]. Furthermore, the efficacy of VTE prophylaxis in patients with COVID-19 is poorly documented [15].

In this context, this study aimed to 1) determine the independent predictors of PE; and 2) evaluate whether anticoagulant therapy is effective for PE prevention in severe COVID-19 patients undergoing CTPA imaging.

\section{Methods}

\section{Study design and population}

This was a retrospective, single-centre study in a university tertiary care hospital in Besançon, France, with a high rate of COVID-19 [16]. We included all patients hospitalised from March 15 to April 16, 2020 with biologically proven COVID-19 pneumonia and CTPA performed due to clinical signs of severity, namely oxygen saturation measured by pulse oximetry $\leqslant 93 \%$ in room air, breathing rate of $\geqslant 30$ breaths $\min ^{-1}$ or rapid clinical worsening [17]. Patients were followed until death or May 5, 2020, even if discharged before this date.

Laboratory confirmation of SARS-CoV-2 was defined as a positive result of real-time reverse transcriptase (RT)-PCR assay of nasal and pharyngeal swabs [18].

VTE prevention in COVID-19 patients comprised anticoagulant therapy at different doses, namely, prophylactic dose (low molecular weight heparin (LMWH): subcutaneous enoxaparin $0.4 \mathrm{mg} \cdot \mathrm{kg}^{-1}$ once daily); or therapeutic dose, with either LMWH (s.c. enoxaparin $1 \mathrm{mg} \cdot \mathrm{kg}^{-1}$ twice daily) or unfractionated heparin (UFH): $80 \mathrm{IU} \cdot \mathrm{kg}^{-1}$ bolus dose followed by $18 \mathrm{IU} \cdot \mathrm{kg}^{-1}$ per hour by continuous infusion to achieve an activated partial thromboplastin time ratio between 1.5 and 2.0; or oral anticoagulant. Management of COVID-19 was at the discretion of the physicians in charge.

In the context of the COVID-19 pandemic, the French national commission for the protection of personal data (CNIL) considers that, for single-centre observational research, the need for information of patients and families is waived. Our protocol followed the ethical guidelines of the declaration of Helsinki and was approved by our institutional review board. Results are reported in accordance with the Strengthening the Reporting of Observational Studies in Epidemiology (STROBE) guidelines [19].

\section{Data collection and variables recorded}

\section{Clinical and biological data}

Baseline characteristics, in-hospital therapies, CTPA findings and adverse events were recorded by research physicians in an anonymous case report form. For each patient, demographic data, medical history and home treatments were collected prospectively at admission. Each patient had a blood sample drawn at admission, and then at least once daily thereafter.

Computed tomography protocol and imaging analysis

Multidetector CTPA was performed on a Revolution CT machine (GE Healthcare, Milwaukee, WI, USA) after intravenous injection of $60 \mathrm{~mL}$ iodinated contrast agent (iomeprol $400 \mathrm{mg}$ iodine. $\mathrm{mL}^{-1}$; Bracco Imaging, Milan, Italy) at a flow rate of $4 \mathrm{~mL} \cdot \mathrm{s}^{-1}$, triggered in the pulmonary trunk.

Affiliations: ${ }^{1}$ Dept of Cardiology, University Hospital, Besançon, France. ${ }^{2}$ Infectious and Tropical Diseases Unit, University Hospital, Besançon. France. ${ }^{3}$ UMR CNRS 6249 Chrono-Environnement, University of FrancheComte, Besancon, France. ${ }^{4}$ Dept of Radiology, Besançon University Hospital, Besançon, France. ${ }^{5}$ Research Unit EA3920, University of Franche-Comte, Besancon, France. ${ }^{6}$ Medical Intensive Care Unit, University Hospital, Besançon, France. ${ }^{7}$ Anesthesia and Surgical Intensive Care Unit, University Hospital, Besançon, France. ${ }^{8}$ Hematology Unit, University Hospital, Besançon, France. ${ }^{9}$ INSERM UMR 1098, University of FrancheComte, Besancon, France. ${ }^{10}$ Virology Laboratory, Úniversity Hospital, Besançon, France. ${ }^{11}$ Both authors contributed equally.

Correspondence: Nicolas Meneveau, Dept of Cardiology, University Hospital Jean Minjoz, 3 Boulevard Fleming, 25000 Besancon, France. E-mail: nicolas.meneveauduniv-fcomte.fr 
Imaging results were reviewed by two chest radiologists. Readers were blinded to clinical and biological features. Readers were asked to assess the COVID-19 pattern by quantitative visual CT evaluation, which consisted of grading acute COVID-19 related lung inflammatory lesions for each lobe, scored as 0 (0\%), 1 (1-25\%), 2 (26$50 \%), 3(51-75 \%)$ or 4 (76-100\%). The total severity score was reached by summing the five lobe scores [20].

In addition, readers were asked to detect presence or absence of PE on CTPA, defined as a filling defect within pulmonary vessels [21]. When PE was present, readers reported extent and topography of PE, signs of right ventricular (RV) dysfunction (i.e. enlargement of the pulmonary artery $>35 \mathrm{~mm}$, abnormal position of the interventricular septum, right ventricular dilation (defined by a RV/left ventricular ratio $>1$ measured in the transverse or four-chamber view on CTPA [22]) or the presence of pulmonary infarction). In case of discordance between readers, scans were re-assessed to reach consensus.

\section{Study objectives}

The primary objective was to determine independent predictors of PE in COVID-19 patients with clinical signs of severity who underwent CTPA. The secondary objective was to evaluate whether anticoagulant therapy is effective for PE prevention in these patients.

\section{Statistical analysis}

Continuous variables are expressed as mean $\pm \mathrm{SD}$ or median (interquartile range (IQR)) as appropriate. Categorical variables are expressed as number (\%). Unadjusted differences between patients with and without CTPA-confirmed PE were compared using the Chi-squared or t-test, as appropriate. To identify independent predictors of CTPA-confirmed PE in severe COVID-19 patients, we used Firth penalised maximum likelihood estimates to adjust our cohort study, which includes a small sample size $(\mathrm{n}=162)$, to overcome the substantial bias linked to logistic regression [23].

Multivariable models included site of care (conventional ward versus intensive care unit (ICU)) as a random effect to account for patient clustering within departments. In multivariable models, continuous covariates were categorised per quartile. All variables with a p-value $<0.10$ by univariate analysis were included in multivariable analysis. Linearity of significant covariates in the multivariable model was verified before computing the receiver operating characteristic (ROC) curve to determine the optimal cut-off value. The multivariable model was then repeated, including independent continuous variables dichotomised based on ROC curve results. The accuracy of the multivariable models was assessed by 1) global model fit (Akaike information criteria and Bayes information criteria); 2) discrimination, using Harrell's C-statistic index; and 3) calibration, by visually plotting the mean of model-predicted CTPA-confirmed PE in each decile against observed PE.

All tests were two-sided. A p-value $<0.05$ was considered significant. Analyses were performed using SAS 9.4 (SAS Institute, Cary, NC, USA).

\section{Results}

\section{Study population}

From March 15 to April 16, 2020, 349 patients were admitted to our tertiary care facility with a diagnosis of COVID-19. In total, 162 (46.4\%) out of 349 patients presented clinical signs of COVID-19 related severity, prompting the physician in charge to prescribe CTPA; 94 (58.0\%) of these were in conventional wards and $68(42.0 \%)$ were in the ICU; 48 (29.6\%) of CTPA procedures were performed at admission, and $114(70.4 \%)$ during the hospital stay. The 162 patients with CTPA imaging comprised the eligible study cohort. Overall, 44 (27.2\%) had PE on CTPA, including 15 (16.0\%) out of 94 in conventional wards, and 29 (42.6\%) out of 68 in the ICU (figure 1). Nine (20.5\%) PEs were diagnosed at admission and 35 (79.6\%) during the hospital stay (supplementary figure S1). The median (IQR) time from hospital admission to CTPA was $5.0(0.0-8.3)$ days.

Table 1 summarises the demographic and clinical characteristics of the population at admission. Mean \pm SD age was $65.57 \pm 13$ years; $67.3 \%$ (95\% CI 59.5-75.5\%) were male. The most common comorbidities were hypertension, dyslipidemia, obesity, diabetes mellitus and a history of any heart disease. There were no significant differences between patients with versus without PE, except more males had PE. Factors known to be associated with an increased risk of VTE, such as history of VTE, cancer and recent surgery did not differ between groups (table 1).

There was no difference in home treatment between groups. 13 (8.0\%, 95\% CI 4.3-13.3\%) patients were taking anticoagulant therapy at admission for a history of VTE or atrial fibrillation (table 1).

In total, during follow-up, 21 (13.0\%, 95\% CI 8.2-19.2\%) out of 162 patients died; 123 (75.9\%, 95\% CI 68.6-82.3\%) out of 162 were discharged alive; and the remaining 18 (11.1\%, 95\% CI 6.7-17.0\%) patients 


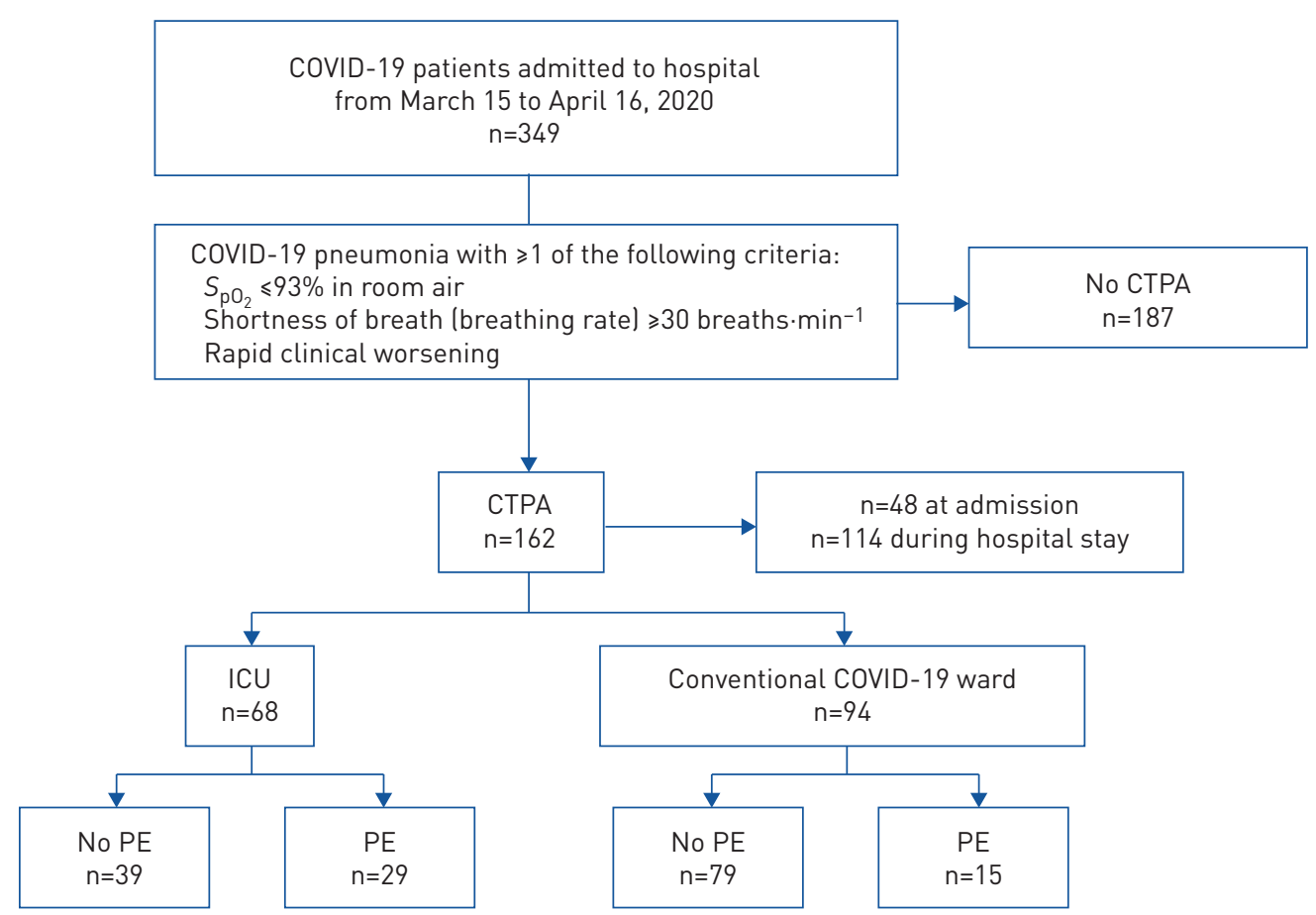

FIGURE 1 Flowchart of the study population. COVID-19: coronavirus disease 2019; $S_{\mathrm{po2}}$ : oxygen saturation measured by pulse oximetry; CTPA: computed tomography pulmonary angiography; ICU: intensive care unit; PE: pulmonary embolism.

were still alive and in hospital on May 5, 2020. The median duration of follow-up of the 162 individuals was 38.0 (33.8-42.3) days, corresponding to 6156 person-days of COVID-19 exposure.

\section{CTPA findings}

CTPA findings are presented in table 2. 25 (56.8\%, 95\% CI 41.0-71.6\%) out of 44 patients had bilateral PE (figure 2). 25 (56.8\%, 95\% CI 41.0-71.6\%) patients had segmental PE. The rate of RV dysfunction in the PE patient group was $15.9 \%$ (95\% CI 6.6-30.1\%).

Regarding the COVID-19 related CT pattern, half of the patients had $>50 \%$ affected lung parenchyma. There was an unadjusted difference in the extent of COVID-19 related CT abnormality between patients with and without PE $(p=0.001)$, whereby forms with $>50 \%$ extension were predominantly found in patients with PE.

\section{Biological parameters}

Unadjusted comparisons of biological results from blood samples drawn on the day of CTPA are summarised in table 3. The median D-dimer level was 4.1 times higher in the PE group compared to patients without PE (5364 (2928-12275) ng. $\mathrm{mL}^{-1}$ versus $1310(800-2335) \mathrm{ng} \cdot \mathrm{mL}^{-1}$, respectively). The rate of liver dysfunction, as assessed by alanine aminotransferase, $\gamma$-glutamyl transferase (GT) and alkaline phosphatase values was higher in those with $\mathrm{PE}$ versus those without. Cardiac troponin I levels were also higher in the PE group $\left(0.018(0.010-0.230) \mu \mathrm{g} \cdot \mathrm{L}^{-1}\right.$ versus $\left.0.010(0.005-0.034) \mu \mathrm{g} \cdot \mathrm{L}^{-1} ; \mathrm{p}=0.003\right)$, whereas there was no difference in brain natriuretic peptide levels between groups. Arterial blood gas analysis revealed hypercapnia associated with elevated bicarbonate $\left(\mathrm{HCO}^{-}\right)$in the $\mathrm{PE}$ group.

\section{VTE prophylaxis during the hospital course}

Overall, $141(87.0 \%, 95 \%$ CI $80.8-91.8 \%)$ out of 162 patients received anticoagulant therapy initiated at admission, including 85.1\% (95\% CI 78.1-90.5\%) with LMWH, 7.8\% (95\% CI 4.0-13.5\%) with UFH and 7.1\% (95\% CI 3.5-12.7\%) with oral anticoagulant. 20.5\% (95\% CI 9.8-35.4\%) of PE patients did not receive any anticoagulation therapy compared with $10.2 \%$ (95\% CI 5.4-17.1\%) of those without PE (unadjusted $\mathrm{p}=0.083$ ). The distribution of preventive and curative anticoagulant doses did not differ between groups: among those without PE, 68.8\% (95\% CI 59.1-77.5\%) received a preventive dose, and 13.2\% (95\% CI 7.4-21.2\%) received a curative dose, versus $85.7 \%$ (95\% CI 77.6-91.7\%) and 14.3\% (95\% CI 
TABLE 1 Baseline characteristics of the study population of coronavirus disease 2019 patients who underwent computed tomography pulmonary angiography

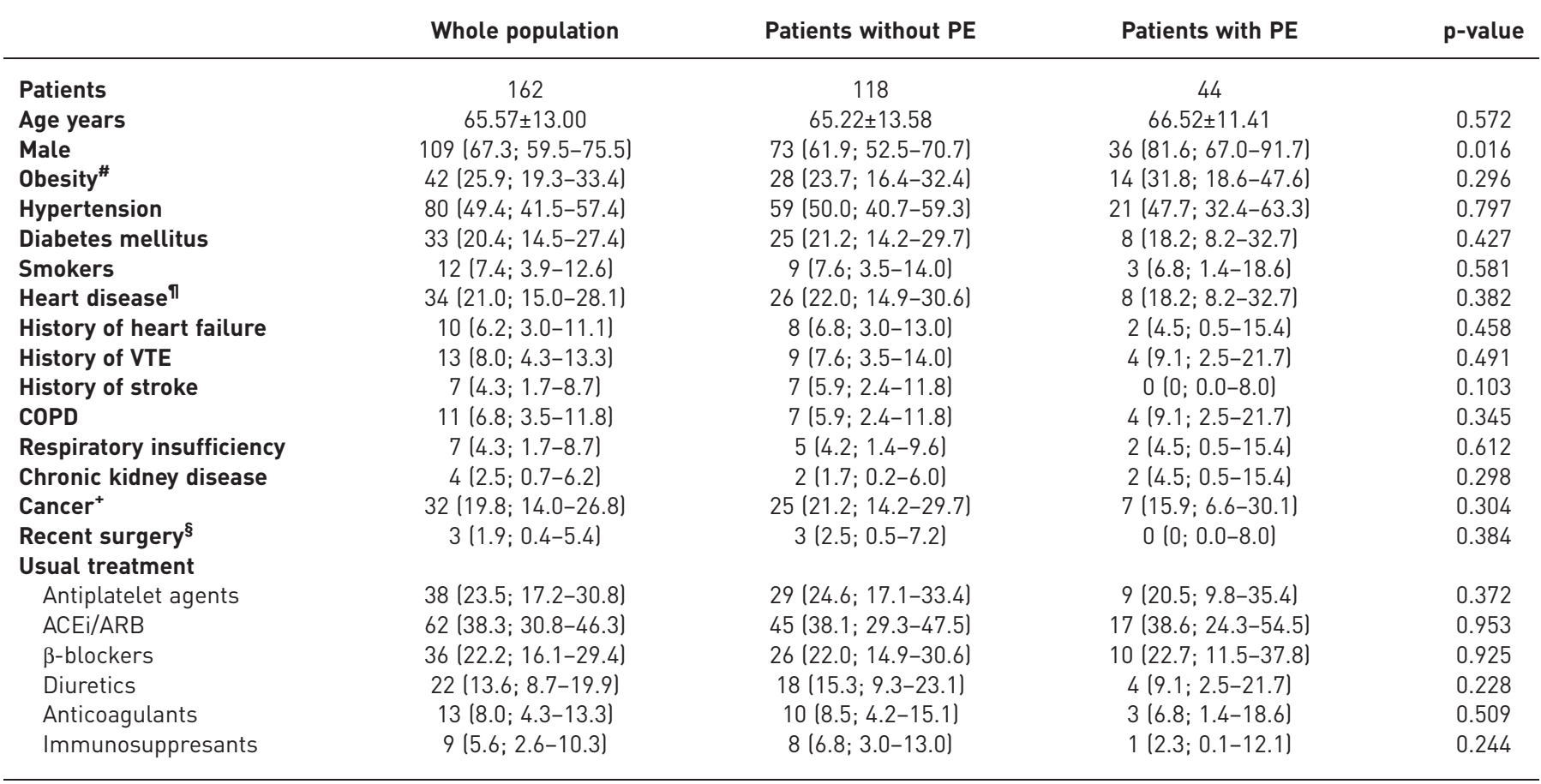

Data are presented as $\mathrm{n}$, mean \pm SD or $\mathrm{n}(\% ; 95 \% \mathrm{CI})$, unless otherwise stated. PE: pulmonary embolism; VTE: venous thromboembolism; ACEi: angiotensin-converting enzyme inhibitor; ARB: angiotensin-receptor blocker. ${ }^{\#}$ : defined as body mass index $>30 \mathrm{~kg} \cdot \mathrm{m}^{-2}$; ${ }^{\text {ๆ }}$ : defined as any history of coronary artery disease, valvular heart disease, arrhythmia or dilated or hypertrophic cardiomyopathy; ${ }^{+}$: active or prior; ${ }^{\S}$ : defined as surgery within the previous 30 days.

8.3-22.4\%), respectively, among patients with PE ( $\mathrm{p}=0.535)$. The distribution of the different anticoagulant regimens is displayed in figure 3.

Predictors of PE in severe COVID-19 patients

By univariate analysis using the Firth penalised likelihood estimator, male sex, D-dimer, arterial carbon dioxide tension, $\mathrm{HCO}^{-}$, blood urea, white blood cell count, extent of COVID-19 related CT scan abnormalities, alkaline phosphatase, $\gamma$-GT, hospitalisation in the ICU (versus conventional COVID ward)

\begin{tabular}{|c|c|c|c|c|}
\hline & Whole population & Patients without PE & Patients with PE & $\mathrm{p}$-value \\
\hline Patients & 162 & 118 & 44 & \\
\hline \multicolumn{5}{|c|}{ Extent of COVID-19 } \\
\hline $0(0 \%)$ & $13(8.0 ; 4.3-13.3)$ & $8(6.8 ; 3.0-13.0)$ & $5(11.4 ; 3.8-24.6)$ & 0.001 \\
\hline $1(1-25 \%)$ & 53 (32.7; 19.3-48.5) & $43(36.4 ; 27.7-45.8)$ & $10(22.7 ; 11.5-37.8)$ & \\
\hline $2(25-50 \%)$ & $48(29.6 ; 22.7-37.3)$ & $41(34.7 ; 26.2-44.0)$ & $7(15.9 ; 6.6-30.1)$ & \\
\hline $3(50-75 \%)$ & $33(20.4 ; 14.5-27.4)$ & $21(17.8 ; 11.4-25.9)$ & $12(27.3 ; 15.0-42.8)$ & \\
\hline 4 (>75\%) & $15(9.3 ; 2.6-21.9)$ & $5(4.2 ; 1.4-9.6)$ & $10(22.7 ; 11.5-37.8)$ & \\
\hline \multicolumn{5}{|l|}{ Extent of emboli } \\
\hline Bilateral & & & $25(56.8 ; 41.0-71.6)$ & \\
\hline \multicolumn{5}{|c|}{ Topography of emboli } \\
\hline Proximal & & & $1(2.3 ; 0.1-12.1)$ & \\
\hline Lobar & & & $16(36.4 ; 22.4-52.3)$ & \\
\hline Segmental & & & $25(56.8 ; 41.0-71.6)$ & \\
\hline \multicolumn{5}{|l|}{ RV function } \\
\hline RV/LV ratio >1 & & & $7(15.9 ; 6.6-30.1)$ & \\
\hline
\end{tabular}

Data are presented as $\mathrm{n}$ or $\mathrm{n}(\% ; 95 \% \mathrm{CI})$, unless otherwise stated. PE: pulmonary embolism; COVID-19: coronavirus disease 2019; RV: right ventricle; LV: left ventricle. 

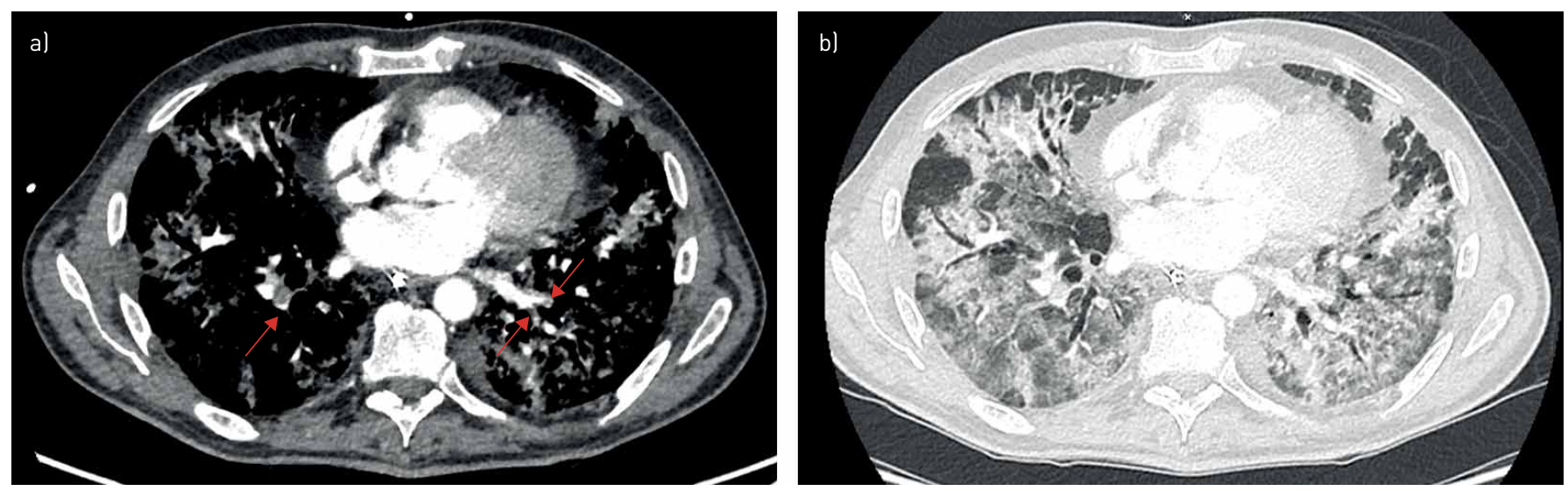

FIGURE 2 Computed tomography pulmonary angiography (CTPA) showing severe coronavirus disease 2019 (COVID-19) pattern and bilateral pulmonary embolism (PE). CTPA of a 54-year-old male with severe acute respiratory syndrome coronavirus 2 (SARS-CoV-2) infection, 23 days after symptom onset and 17 days after admission to the intensive care unit. CTPA was performed because of severe hypoxaemia despite invasive mechanical ventilation, and showed, in addition to a severe COVID-19 computed tomography pattern, bilateral acute PE of segmental location. a) mediastinum window: presence of acute PE as a filling defect inside the left superior lobe pulmonary artery, segmental-subsegmental division of the lingula (red arrows); b) parenchymal window: COVID-19 CT pattern with peripheral ground-glass opacities associated with areas of consolidation.

and any anticoagulant therapy were all significantly associated with PE diagnosis on CTPA (supplementary table S1).

By multivariable analysis, D-dimer level and the lack of any anticoagulant therapy were significantly associated with the occurrence of CTPA-confirmed PE (OR 4.0 (95\% CI 2.4-6.7) per additional quartile of D-dimer and OR 4.5 (95\% CI 1.1-7.4), respectively) (figure 4).

Linearity was verified for D-dimers in the study population (test of linearity $<0.001$ ). ROC curve analysis determined the optimal cut-off value of $2590 \mathrm{ng} \cdot \mathrm{mL}^{-1}$ for the D-dimer level to predict CTPA-confirmed

TABLE 3 Biological results from blood samples drawn at the time of computed tomography pulmonary angiography

Patients (n1/n2)

All

Patients without PE (n1)

Patients with PE (n2)

p-value

Haemostasis

D-dimers $\mathrm{ng} \cdot \mathrm{mL}^{-1}$

Complete blood count

Leukocytes $\times 10^{9}$ cells. $\mathrm{L}^{-1}$

Lymphocytes $\times 10^{9}$ cells $\cdot \mathrm{L}^{-1}$

Haemoglobin $\mathrm{g} \cdot \mathrm{dL}^{-1}$

Platelet count $\times 10^{9}$ cells. $\mathrm{L}^{-1}$

\section{Biochemistry}

Procalcitonin $\mathrm{ng} \cdot \mathrm{mL}^{-1}$

Bicarbonates $\mathrm{mmol} \cdot \mathrm{L}^{-1}$

Urea $\mathrm{mmol} \cdot \mathrm{L}^{-1}$

Creatininaemia $\mu \mathrm{mol} \cdot \mathrm{L}^{-1}$

ASAT IU. $L^{-1}$

ALAT IU.L $\mathrm{L}^{-1}$

$\gamma$-Glutamyl transferase IU.L $\mathrm{L}^{-1}$

Alkaline phosphatase IU. $\mathrm{L}^{-1}$

C-reactive protein $\mathrm{mg} \cdot \mathrm{L}^{-1}$

Troponin Ic $\mu \mathrm{g} \cdot \mathrm{L}^{-1}$

BNP $\mathrm{ng} \cdot \mathrm{mL}^{-1}$

\section{Blood gas}

$\mathrm{pH}$

$\mathrm{PaCO}_{2} \mathrm{kPa}$

$\mathrm{PaO}_{2} \mathrm{kPa}$

Lactates $\mathrm{mmol} \cdot \mathrm{L}^{-1}$

$122(80 / 42)$
$152(112 / 40)$
$59(49 / 10)$
$153(113 / 40)$
$153(113 / 40)$
$102(76 / 26)$
$159(116 / 43)$
$158(115 / 43)$
$157(115 / 42)$
$135(98 / 37)$
$147(110 / 37)$
$130(92 / 38)$
$130(92 / 38)$
$158(114 / 44)$
$154(111 / 43)$
$150(112 / 38)$

$115(76 / 39)$

$115(76 / 39)$

$115(76 / 39)$

$115(76 / 39)$

$\begin{array}{cc}1920(1068-4020) & 1310(800-2335) \\ 7.45(5.80-10.20) & 6.70(5.53-9.48) \\ 1.00 \pm 0.43 & 0.94 \pm 0.42 \\ 12.45 \pm 2.17 & 12.65 \pm 2.11 \\ 266(201.5-391) & 246(188-387) \\ & \\ 0.23(0.13-0.42) & 0.26(0.13-0.43) \\ 24.00(21.70-27.40) & 23.55(21.20-25.90) \\ 5.90(4.48-9.43) & 5.70(4.20-7.90) \\ 71(57-90) & 72(58-90) \\ 46(34-69) & 44(34-67) \\ 37(24-58) & 36(23-53) \\ 91(45-192) & 79(40-146) \\ 100(68-160) & 84(66-135) \\ 115(69-196) & 114(66-193) \\ 0.010(0.010-0.040) & 0.010(0.005-0.034) \\ 36(12-104) & 35(12-91)\end{array}$

$7.45(7.40-7.49)$

$4.71(4.24-6.81)$

$10.20(8.80-12.35)$

$1.40(1.20-1.70)$
$7.45(7.40-7.49)$

$4.67(4.18-5.62)$

$9.95(8.70-11.70)$

$1.40(1.20-1.70)$

$\begin{array}{cc}5364(2928-12275) & <0.001 \\ 8.92(6.55-13.85) & 0.002 \\ 1.28 \pm 0.38 & 0.023 \\ 11.89 \pm 2.24 & 0.056 \\ 343(247-397) & 0.006 \\ & \\ 0.17(0.13-0.27) & 0.130 \\ 26.60(23.60-32.00) & <0.001 \\ 8.90(5.10-13.50) & 0.001 \\ 70(52-87) & 0.754 \\ 53(39-72) & 0.194 \\ 54(30-76) & 0.010 \\ 160(78-273) & 0.002 \\ 131(82-248) & 0.005 \\ 121(72-198) & 0.861 \\ 0.018(0.010-0.230) & 0.003 \\ 43(13-189) & 0.410 \\ & \\ 7.44(7.40-7.49) & 0.859 \\ 5.96(4.45-7.44) & 0.021 \\ 10.60(8.80-13.10) & 0.268 \\ 1.30(1.10-1.60) & 0.198\end{array}$

Data are presented as $n$, mean \pm SD or median (interquartile range), unless otherwise stated. PE: pulmonary embolism; ASAT: aspartate aminotransferase; ALAT: alanine aminotransferase; BNP: brain natriuretic peptide; $P_{\mathrm{aCO}_{2}}$ : arterial carbon dioxide tension; $P_{\mathrm{aO}}$ : arterial oxygen tension. 


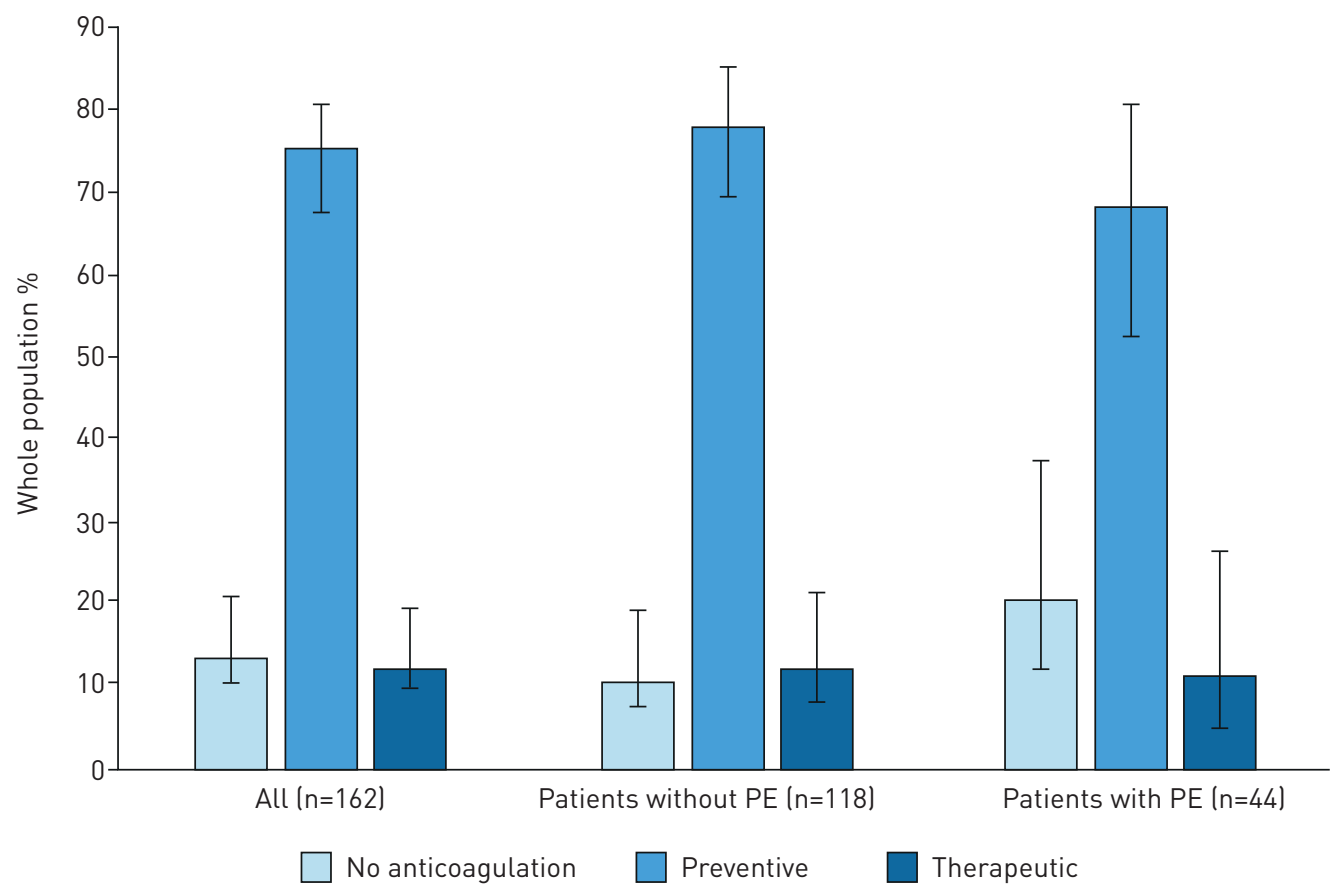

FIGURE 3 Distribution of the different anticoagulant regimens in the whole study population and by group of patients with and without computed tomography pulmonary angiography-confirmed pulmonary embolism (PE).

PE in severe COVID-19 patients with high accuracy: AUC 0.88 (95\% CI 0.809-0.932, p<0.001); Youden index 0.6708; sensitivity $83.3 \%$ (95\% CI 68.6-93.0\%); specificity $83.8 \%$ (95\% CI $73.8-91.1 \%$ ); positive predictive value $72.9 \%$ (95\% CI 61.7-81.8\%); and negative predictive value $90.5 \%$ (95\% CI $82.9-95.0 \%$ ) (figure 5).

Patients with D-dimers above the cut-off of $2590 \mathrm{ng} \cdot \mathrm{mL}^{-1}$ accounted for $36.0 \%$ (95\% CI 27.5-45.2\%) of the overall population, $42.6 \%$ (95\% CI 30.7-55.2\%) of patients in ICU and $15.9 \%$ (95\% CI 9.2-24.9) of patients in conventional COVID wards. When analysed in multivariable analysis as a binary variable, using the ROC-defined cut-off, a D-dimer level $>2590 \mathrm{ng} \cdot \mathrm{mL}^{-1}$ was found to be a significant predictor of PE (OR 16.9, 95\% CI 6.3-45.0). Additionally, the lack of any anticoagulation was significantly associated with PE (OR 4.0, 95\% CI 1.1-14.2) (figure 4). The accuracy of both multivariable models was good, as assessed by global model fit, discrimination and visual calibration, with predicted risks and their confidence intervals distributed around the observed risk of CT-scan confirmed PE (figure 4) (supplementary figures S2 and S3).

\section{Discussion}

To the best of our knowledge, this study is the first to identify independent predictors of the occurrence of PE, from a cohort of 162 COVID-19 patients with clinical signs of severity. Our data show that PE was frequent in these patients, while elevated D-dimer levels and the absence of any anticoagulant therapy were both found to be independent predictors of PE. Specifically, a D-dimer level $>2590 \mathrm{ng} \cdot \mathrm{mL}^{-1} \mathrm{was}$ associated with a 17 -fold increase, and lack of anticoagulation with a four-fold increase in the risk of PE. Furthermore, the prevalence of PE remained high in this patient population, regardless of the type of anticoagulant strategy used. These data plead in favour of wider screening for PE by performing D-dimer guided CTPA in COVID-19 patients with signs of clinical severity.

The rate of PE observed in our study in hospitalised COVID-19 patients with clinical signs of severity is high $(27.2 \%)$, and in line with rates reported in other studies. [6, 8-10]. The increased thromboembolic risk in COVID-19 patients could be enhanced by a procoagulant state generated by the severity of the infection [2], the magnitude of the inflammatory response [3] and liver dysfunction [24-26]. The most frequently reported biological anomalies in COVID-19 patients include elevations of inflammatory markers such as C-reactive protein, D-dimers, ferritin and interleukin-6 [27, 28]. In our population, $\mathrm{D}$-dimer levels were four times higher in PE patients than in non-PE patients. This elevation of D-dimer levels has been established as being associated with severity of disease and mortality in COVID-19 patients 


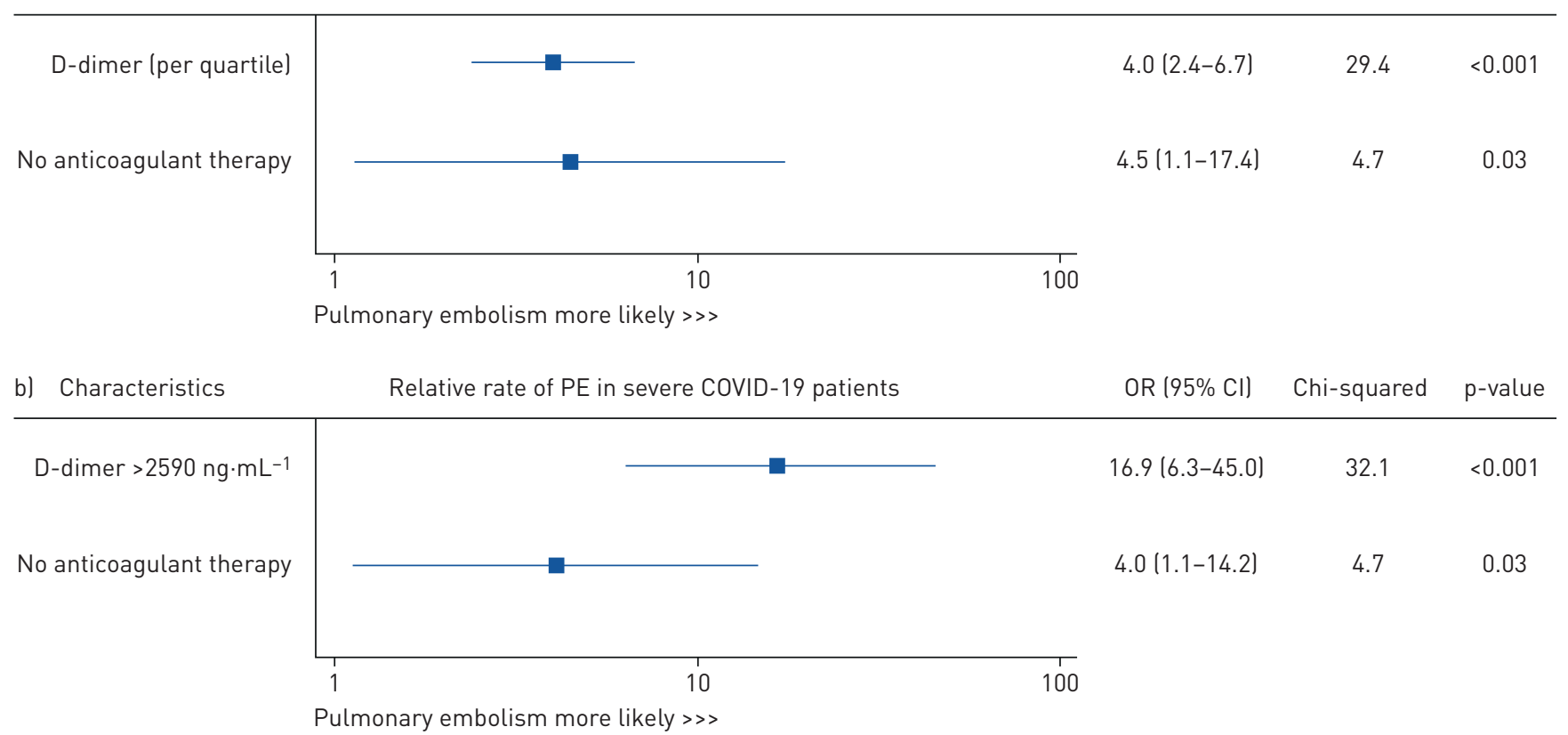

FIGURE 4 Independent predictors of in-hospital computed tomography pulmonary angiography-confirmed pulmonary embolism (PE) in severe coronavirus disease 2019 (COVID-19) patients, using the Firth penalised likelihood estimator. a) Multivariable model including D-dimer analysed by quartile, D-dimer level (model fit: Akaike information criteria 99.1 and Bayes information criteria 133.1 for global model fit, and Harrell's C-statistic index 0.91 for discrimination). b) Multivariable model including D-dimer as a binary variable defined by receiver operating characteristic curve analysis (model fit: Akaike information criteria 104.6 and Bayes information criteria 138.4 for global model fit, and Harrell's C-statistic index 0.90 for discrimination).

and should be considered to reflect activation of the coagulation system in this setting $[3,9,28-30]$. In a Chinese study of 183 patients with COVID-19, D-dimer levels were 3.5 times higher in patients who died compared to patients who survived [3]. Similarly, COVID-19 patients with increased D-dimer concentration at admission $\left(>1000 \mathrm{ng} \cdot \mathrm{mL}^{-1}\right)$ were reported to have an 18-fold higher risk of in-hospital mortality than those with normal D-dimer levels [28]. Our data confirm this finding, and strengthen the evidence underpinning the relationship between elevated D-dimers and the risk of PE in COVID-19 patients [1]. The threshold for D-dimers that we identified, i.e. $2590 \mathrm{ng} \cdot \mathrm{mL}^{-1}$, was the strongest independent predictor of $\mathrm{PE}$ in our population. To the best of our knowledge, this study is the first to identify a threshold for D-dimer levels that is an independent predictor of PE in COVID-19 patients after

FIGURE 5 Receiver operating characteristic curve identifying the cut-off value of $D$-dimer predictive of occurrence of pulmonary embolism. AUC: area under the curve.

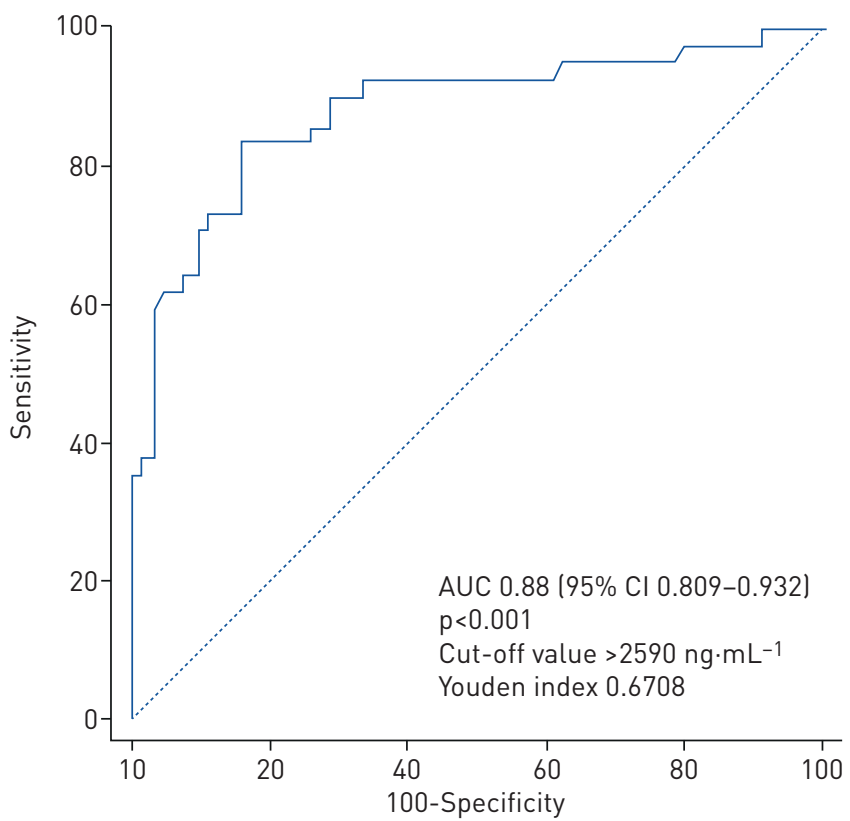


multivariable adjustment. Therefore, particular attention should be paid to search for potential PE in patients with clinical criteria of severity and with a D-dimer level $>2590 \mathrm{ng} \cdot \mathrm{mL}^{-1}$, since PE is a life-threatening but potentially treatable condition [22].

Our data confirm that anticoagulation for the prevention of VTE is absolutely crucial in the most severe COVID-19 patients, and administration of anticoagulants reduces the risk of PE four-fold. The relatively high proportion of patients who did not receive preventive anticoagulation in our study can probably be explained by the fact that $20.5 \%$ of patients had a diagnosis of PE at admission and were thus admitted directly from home with no anticoagulation in place. A recently published short report including 2773 COVID-19 patients showed that longer duration of anticoagulant treatment was associated with a reduced risk of in-hospital mortality (adjusted hazard ratio 0.86 per day, 95\% CI 0.82-0.89) [31]. In our study, regardless of the regimen used, the prevalence of PE remained high. However, our data do not enable us to determine the most appropriate prophylactic strategy, or to answer the question of whether curative doses are more effective than preventive doses in averting PE. This question was the subject of some debate during the development of a recent consensus on the prevention, antithrombotic therapy and follow-up of thrombotic or thromboembolic disease in COVID-19 patients [15]. Indeed, in this document, the authors were unable to reach consensus on the optimal dosing of anticoagulant therapy, stipulating that "the majority of panel members consider prophylactic anticoagulation, although a minority consider intermediate-dose or therapeutic dose to be reasonable" [15]. There is thus a compelling need for prospective studies investigating the optimal dosing in patients with severe COVID-19. In practice, pluridisciplinary discussion is warranted to evaluate the patient's haemorrhagic risk, and to weigh it against the risk of VTE. In light of our results, this point is of paramount importance in COVID-19 patients with severe forms of disease and evidence of activation of the coagulation system (e.g. elevated D-dimers) in whom prophylactic anticoagulant treatment appears to be essential for the prevention of PE. This is in accordance with a study of 449 patients with severe COVID-19 from China, where a reduction in mortality of $\sim 20 \%$ was observed with heparin treatment in patients who had D-dimers $>3000 \mathrm{ng} \cdot \mathrm{mL}^{-1}$ (six times the upper limit of normal) [32]. Furthermore, a recent expert panel document from the Global COVID-19 Thrombosis Collaborative Group postulates that LMWH and UFH may have anti-inflammatory and antiviral properties [33], although there is no established link between these properties and the course of COVID-19 disease.

Although current guidelines from professional societies of radiology recommend performing noncontrast chest CT to assess the COVID-19 pattern and its extension [11-13], our data plead in favour of a wider screening strategy for PE by performing CTPA in COVID-19 patients who have signs of clinical severity and D-dimer levels $>2590 \mathrm{ng} \cdot \mathrm{mL}^{-1}$. Indeed, the utility of CTPA over noncontrast CT is that it covers the whole spectrum of possible COVID-19 related complications, including COVID-19 related lung injuries, parenchymal bacterial infection, pleural effusion and pneumothorax, as well as enabling diagnosis of PE thanks to contrast injection. The high prevalence of $\mathrm{PE}$ in patients receiving anticoagulation is an additional argument in favour of this approach. The D-dimer cut-off identified in this study could be used to guide CTPA use, particularly in patients at risk of contrast-induced acute kidney injury. Indeed, it has recently been shown that acute kidney injury was associated with unfavourable outcome in hospitalised COVID-19 patients $[34,35]$. Further prospective studies with larger sample sizes are warranted to externally validate the optimal D-dimer cut-off value related to PE in acutely ill hospitalised COVID-19 patients.

Our study has some limitations. Firstly, it is a retrospective study from a single centre, and we cannot exclude the possible presence of unmeasured confounders. The sample size is relatively small, although it is the largest series of COVID-19 patients undergoing CTPA reported to date. Only patients undergoing CTPA were included, and it is thus possible that the actual rate of PE was even higher than reported here. The selection of patients to undergo CTPA was based on clinical criteria of severity that may be debatable. Furthermore, most patients did not have compression ultrasonography screening during the study period. Our data do not make it possible to identify the most appropriate prophylactic anticoagulation strategy, and notably, do not answer the question of whether curative anticoagulation is more effective than preventive anticoagulation in averting PE. Finally, transthoracic echocardiographic data was not recorded in the present study to assess RV function in COVID-19 associated PE patients. However, current guidelines stipulate that CTPA is a validated alternative for the evaluation of RV dysfunction in acute PE [22].

Despite these limitations, this study is the first to identify independent predictors of $\mathrm{PE}$, using robust statistical methods, and to report exhaustive biological and radiological findings as well as details of anticoagulant therapy in COVID-19 patients at the peak of the epidemic in France. 
patients. These data strengthen the evidence base in favour of systematic anticoagulation, and suggest wider use of D-dimer guided CTPA to screen for PE in acutely ill hospitalised patients with COVID-19. The optimal dosing of anticoagulants remains unknown and warrants further prospective investigation.

Conflict of interest: None declared.

\section{References}

1 Cui S, Chen S, Li X, et al. Prevalence of venous thromboembolism in patients with severe novel coronavirus pneumonia. J Thromb Haemost 2020; 18: 1421-1424.

2 Fan BE, Chong VCL, Chan SSW, et al. Hematologic parameters in patients with COVID-19 infection. Am J Hematol 2020; 95: E131-E134.

3 Tang N, Li D, Wang X, et al. Abnormal coagulation parameters are associated with poor prognosis in patients with novel coronavirus pneumonia. J Thromb Haemost 2020; 18: 844-847.

4 Lew TW, Kwek TK, Tai D, et al. Acute respiratory distress syndrome in critically ill patients with severe acute respiratory syndrome. JAMA 2003; 290: 374-380.

5 Zhang Y, Xiao M, Zhang S, et al. Coagulopathy and antiphospholipid antibodies in patients with Covid-19. $N$ Engl J Med 2020; 382: e38.

6 Klok FA, Kruip M, van der Meer NJM, et al. Incidence of thrombotic complications in critically ill ICU patients with COVID-19. Thromb Res 2020; 191: 145-147.

7 Klok FA, Kruip M, van der Meer NJM, et al. Confirmation of the high cumulative incidence of thrombotic complications in critically ill ICU patients with COVID-19: an updated analysis. Thromb Res 2020; 191: 148-150.

8 Poissy J, Goutay J, Caplan M, et al. Pulmonary embolism in COVID-19 patients: awareness of an increased prevalence. Circulation 2020; 142: 184-186.

9 Léonard-Lorant I, Delabranche X, Séverac F, et al. Acute pulmonary embolism in patients with COVID-19 at CT angiography and relationship to D-dimer levels. Radiology 2020; 296: E189-E191.

10 Llitjos JF, Leclerc M, Chochois C, et al. High incidence of venous thromboembolic events in anticoagulated severe COVID-19 patients. J Thromb Haemost 2020; 18: 1743-1746.

11 Revel MP, Parkar AP, Prosch H, et al. COVID-19 patients and the radiology department - advice from the European Society of Radiology (ESR) and the European Society of Thoracic Imaging (ESTI). Eur Radiol 2020; 30: 4903-4909.

12 Simpson S, Kay FU, Abbara S, et al. Radiological Society of North America Expert consensus statement on reporting chest CT findings related to COVID-19. Endorsed by the Society of Thoracic Radiology, the American College of Radiology, and RSNA. J Thorac Imaging 2020; 35: 219-227.

13 Rubin GD, Ryerson CJ, Haramati LB, et al. The role of chest imaging in patient management during the COVID-19 pandemic: a multinational consensus statement from the Fleischner Society. Chest 2020; 158: 106-116.

14 Grillet F, Behr J, Calame P, et al. Acute pulmonary embolism associated with COVID-19 pneumonia detected by pulmonary CT angiography. Radiology 2020; 296: E186-E188.

15 Bikdeli B, Madhavan MV, Jimenez D, et al. COVID-19 and thrombotic or thromboembolic disease: implications for prevention, antithrombotic therapy, and follow-up. J Am Coll Cardiol 2020; 75: 2950-2973.

16 Santé Publique France. Standardized Level of Excess Mortality, All Ages, 2020 Week 14. Géodes - Geo Data in Public Health Database. 2020. https://geodes.santepubliquefrance.fr/\#c=indicator\&f=0\&i=covid_xcdc_public.cat_ zscore\&s=2020-S14\&t=a01\&view=map2 Date last accessed: April 26, 2020.

17 Metlay JP, Waterer GW, Long AC, et al. Diagnosis and treatment of adults with community-acquired pneumonia. An official clinical practice guideline of the American Thoracic Society and Infectious Diseases Society of America. Am J Respir Crit Care Med 2019; 200: e45-e67.

18 World Health Organization (WHO). Clinical Management of Severe Acute Respiratory Infection (SARI) When COVID-19 Disease is Suspected. Interim Guidance. Geneva, WHO, 2020. www.who.int/publications-detail/ clinical-management-of-severe-acute-respiratory-infection-when-novel-coronavirus-(ncov)-infection-is-suspected Date last accessed: April 23, 2020.

19 von Elm E, Altman DG, Egger M, et al. The Strengthening the Reporting of Observational Studies in Epidemiology (STROBE) statement: guidelines for reporting observational studies. Epidemiology 2007; 18: 800-804.

20 Li K, Fang Y, Li W, et al. CT image visual quantitative evaluation and clinical classification of coronavirus disease (COVID-19). Eur Radiol 2020; 30: 4407-4416.

21 Remy-Jardin M, Remy J, Wattinne L, et al. Central pulmonary thromboembolism: diagnosis with spiral volumetric CT with the single-breath-hold technique - comparison with pulmonary angiography. Radiology 1992; 185: 381-387.

22 Konstantinides SV, Meyer G, Becattini C, et al. 2019 ESC Guidelines for the diagnosis and management of acute pulmonary embolism developed in collaboration with the European Respiratory Society (ERS). Eur Heart J 2020; 41: 543-603.

23 Sun JX, Sinha S, Wang S, et al. Bias reduction in conditional logistic regression. Stat Med 2011; 30: 348-355.

24 Zhang C, Shi L, Wang FS. Liver injury in COVID-19: management and challenges. Lancet Gastroenterol Hepatol 2020; 5: 428-430.

25 Guan WJ, Ni ZY, Hu Y, et al. Clinical characteristics of coronavirus disease 2019 in China. N Engl J Med 2020; 382: $1708-1720$.

26 Huang C, Wang Y, Li X, et al. Clinical features of patients infected with 2019 novel coronavirus in Wuhan, China. Lancet 2020; 395: 497-506.

27 Wang D, Hu B, Hu C, et al. Clinical characteristics of 138 hospitalized patients with 2019 novel coronavirus-infected pneumonia in Wuhan, China. JAMA 2020; 323: 1061-1069.

28 Zhou F, Yu T, Du R, et al. Clinical course and risk factors for mortality of adult inpatients with COVID-19 in Wuhan, China: a retrospective cohort study. Lancet 2020; 395: 1054-1062.

29 Spyropoulos AC, Ageno W, Barnathan ES. Hospital-based use of thromboprophylaxis in patients with COVID-19. Lancet 2020; 395: e75. 
30 Lippi G, Favaloro EJ. D-dimer is associated with severity of coronavirus disease 2019: a pooled analysis. Thromb Haemost 2020; 120: 876-878.

31 Paranjpe I, Fuster V, Lala A, et al. Association of treatment dose anticoagulation with in-hospital survival among hospitalized patients with COVID-19. J Am Coll Cardiol 2020; 76: 122-124.

32 Tang N, Bai $\mathrm{H}$, Chen $\mathrm{X}$, et al. Anticoagulant treatment is associated with decreased mortality in severe coronavirus disease 2019 patients with coagulopathy. J Thromb Haemost 2020; 18: 1094-1099.

33 Bikdeli B, Madhavan MV, Gupta A, et al. Pharmacological agents targeting thromboinflammation in COVID-19: review and implications for future research. Thromb Haemost 2020; 120: 1004-1024.

34 Cheng Y, Luo R, Wang K, et al. Kidney disease is associated with in-hospital death of patients with COVID-19. Kidney Int 2020; 97: 829-838.

35 Ozkok S, Ozkok A. Contrast-induced acute kidney injury: a review of practical points. World J Nephrol 2017; 6: 86-99. 